\title{
¿Qué piensan los profesores sobre los niños con talento académico? Una aproximación a las concepciones de profesores de segundo ciclo de educación básica en Chile*
}

\author{
What do Teachers Think about Children Academically Talented? \\ An Approach to Middle School Teachers Conceptions in Chile
}

Recibido: 27 de diciembre de 2013| Aceptado: 08 de marzo de 2016

\author{
Elsa Piedad Cabrera-Murcia ** \\ Universidad Alberto Hurtado, Chile \\ Cecilia UdAQUIOLA $* * *$ \\ Craighouse School, Chile
}

doi: 10.11144/Javeriana.upsy15-2.ppnt

Para citar este artículo: Cabrera-Murcia, E. P. \& Udaquiola, C. (2016). ¿Qué piensan los profesores sobre los niños con talento académico? Una aproximación a las concepciones de profesores de segundo ciclo de educación básica en Chile. Universitas Psychologica, 15(2), 121-134. http://dx.doi. org/10.11144/Javeriana.upsy15-2.ppnt

* Artículo de investigación. No tuvo financiación. Agradecimientos: Agradecemos a los profesores que participaron activamente en esta investigación, su apoyo en la recolección de información fue relevante para el desarrollo de la misma.

*** Asistente Académico Dirección Docencia, Universidad Alberto Hurtado. Correo electrónico: epcabrer@uc.cl

**** Correo electrónico:ceciliaudaquiola@gmail.com

\section{RES U MEN}

Este artículo describe las concepciones de trece profesores de segundo ciclo de educación básica, de tres colegios de la Región Metropolitana de Chile, en torno a los estudiantes con talento académico y su educación. Se utilizó una metodología cualitativa enmarcada en los principios de la Grounded Theory, y se recolectaron los datos a partir de la aplicación de dilemas y entrevistas semiestructuradas. Los resultados muestran el desarrollo cognitivo, social y emocional como marcadores distintivos en niños con talento; el origen del talento como factor determinante; la graduación del talento como factor diferenciador; los ambientes enriquecidos como factores potenciadores del talento, y el reconocimiento de un sistema educativo que homogeniza la educación y no reconoce las diferencias. El análisis relacional destaca tres fenómenos que ponen en evidencia una concepción más contemporánea del talento académico: el reconocimiento de niños con talento académico en el aula, el rol del profesor en el desarrollo y potenciación del talento académico y la educación como una oportunidad de movilidad social. Para concluir, se reafirma la necesidad de considerar concepciones menos tradicionales, así como la posibilidad de generar instancias formativas en las que la educación de niños con talento sea responsabilidad de todos los profesores. Asimismo, se señala cómo las limitaciones de este estudio dan apertura a nuevas líneas de indagación.

Palabras clave

estudiantes con talento académico; concepciones profesores; origen del talento; factores potenciadores del talento; metodología cualitativa

\section{A B S T R A C T}

This article describes the conceptions of thirteen teachers of Middle School about talented students and their education in three different schools of the Metropolitan Region of Chile. A qualitative methodology framed in the principles of Grounded Theory was used, and data was collected from the application of dilemmas and semi-structured interviews. The results show the cognitive, social and emotional development as distinctive trade in talent students, the origin of talent as a determining factor, graduation talent as a differentiating factor, enriched environments as factors that promote talent, and the recognition of an educational system that homogenize the education and doesn't recognize the differences. The relational analysis highlights the recognition of children with academic talent in the classroom, the teacher's role in the development and enhancement of 
academic talent and education as an opportunity for social mobility as three phenomena that evidence a contemporary talent academic design. In conclusion, reinforces are needed to consider non-traditional conceptions and the possibility to generate training instances in which the education of talent children, is the responsibility of all teachers. It also noted how the limitations of this study provide openness to new lines of research.

Keywords

talented students; teachers conceptions; origin of talent; factors that promote talent; qualitative methodology

\section{Introducción}

La educación de niños con talento es considerada hoy un tema clave en la agenda de las políticas educativas a nivel global. En Europa, sus políticas promueven la atención diferenciada a todos los estudiantes, así como la posibilidad de generar programas de atención a estudiantes talentosos (Sekowski \& Lubianka, 2015). En países como Hong Kong, la reciente reforma educativa da prioridad a potenciar en todas las escuelas el pensamiento crítico, la creatividad y la comunicación, tres habilidades que están en el corazón de sus planteamientos en educación de talentos (Chan \& Yuen, 2015; Education Bureau, 2007). Mientras que en países iberoamericanos — como Argentina, Bolivia, Colombia, España, México, entre otros- se ha explicitado la necesidad de atender a la diversidad de estudiantes que asisten al sistema escolar (MEN, 2006; Ministerio de Educación Argentina, 2006; Ministerio de Educación Bolivia; 2010; SEP, 2003; Benavides, Maz, Castro, \& Blanco, 2004). De manera particular, estas normativas han impactado mayormente la generación de programas educativos de iniciativa privada que brindan oportunidades educativas a niños con talento (Benavides et al., 2004).

En Chile, desde hace una década, se ha ido tomando conciencia que las necesidades educativas especiales también incluyen a los estudiantes con altas capacidades (Cabrera-Murcia, 2011; 2012; García-Cepero, Muñoz, Proestakis, López, \& Guzmán, 2010; García-Cepero, Proestakis, Lillo, López, \& Guzmán, 2012). La atención educativa a esta población de estudiantes ha sido asumida desde seis universidades, distribuidas en diferentes regiones del país, con la implementación de programas de enriquecimiento. Los niños favorecidos con este tipo de programas, "corresponden a estudiantes que poseen capacidades naturales en un grado que sitúa al individuo dentro del rango superior de su grupo de edad, en al menos, un área de las habilidades humanas" (García-Cepero, Muñoz et al, 2010).

Estas iniciativas se enfocan en un grupo particular de niños con talento académico sin considerar concepciones más contemporáneas (Tapper, 2012), como aquellas que enmarcan el talento como una propiedad emergente de factores personales tanto como de condiciones ambientales (Kaufman \& Sternberg, 2008). Si nos posicionamos en esta última, es muy probable que al aula regular asistan niños con talento que al no acceder a los programas de enriquecimiento existentes, y al no ser visibilizados por el sistema escolar, su talento no se promueve ni desarrolla. Esto puede traer como consecuencias la frustración, desmotivación o incluso, a nivel social, el sentir no ser un aporte al bien común (García-Huidobro, 2005). iSon conscientes los profesores de esta realidad en su aula? ¿Cuáles son sus concepciones en relación al tema y cómo abordan la enseñanza de estos niños?

\section{¿Qué entenderemos por concepciones?}

Formar profesores implica generar espacios en los que se reflexione antes, durante y después de este proceso sobre cuáles son las concepciones que construyen durante su formación, fruto de las experiencias obtenidas en su entorno social. Algunos autores apoyan esta premisa, pues afirman que las concepciones de los profesores y su práctica son indisociables del proceso de enseñanza y éstas se manifiestan en forma explícita cuando se le demanda o solicita a la persona que se exprese verbalmente, manifieste sus ideas, discrimine, reflexione o argumente acerca del dominio de un conocimiento (Pozo, 2006; Rodrigo, Rodríguez, \& Marrero, 1993). Podríamos afirmar, por tanto, que éstas juegan un papel clave en cómo los profesores aprenden a interpretar la información que nutre los procesos de enseñanzaaprendizaje y que alimentarán sus prácticas de clase (Pajares, 1992; Pozo, 2006). Hacerlas explícitas se constituye en un punto de partida para mejorar la enseñanza (Vilanova, García, \& Señoriño, 2007). 
Para efectos de esta investigación, hablaremos de concepciones, aludiendo a las construcciones personales de conocimiento en torno a un dominio, que están en un nivel representacional explícito, fruto de experiencias obtenidas con el entorno social y cultural, y que son expresadas cuando una persona las utiliza de forma declarativa para verbalizar, reflexionar, debatir o argumentar (Rodrigo et al., 1993). Consideraremos que estas concepciones reflejan una versión construida, personal, alimentada por las experiencias en interacción con otros y que se explicitan en situaciones de la vida diaria.

¿Qué dicen las investigaciones en el contexto internacional sobre las concepciones en torno a los niños con talento y su educación?

Una revisión de literatura internacional pone en evidencia investigaciones realizadas en distintos países acerca de las concepciones que poseen profesores en ejercicio y en formación sobre la educación de talentos y de los estudiantes talentosos (Chung, Kim, Lee, \& Park, 2013; Jaffri, 2012; Kontoyianni, et al., 2009; Plunkett, 2011; Sumpter \& Sternevik, 2013). Plunkett (2011) exploró las actitudes de estudiantes de pedagogía antes y después de asistir a un semestre electivo en educación de talentos en la universidad. Los participantes mostraron un cambio significativo en las opiniones, comprensión de los superdotados y su educación, así como fuertes lazos entre el conocimiento específico adquirido y el desarrollo de actitudes positivas frente a estudiantes con talento, materializado en un apoyo escolar apropiado.

Kontoyianni et al. (2009) examinó la estructura de las percepciones de profesores de colegios de educación básica en el área de matemáticas. Necesidades del profesor, creencias acerca de la eficacia profesional docente, características del alumno talentoso y los apoyos para satisfacer las necesidades de estos niños son las dimensiones que caracterizan las percepciones de los profesores. En relación a la última, algunos de ellos consideraron la diferenciación como estrategia educativa, mientras que otros hicieron hincapié en que estos niños no necesitan educación especial.
Dichos resultados se relacionan con los señalados por Tallent-Runnels \& Tirri, (1998, citados en Tirri, 2008) en su investigación acerca de las creencias frente a la educación del talento de profesores provenientes de diferentes contextos socioculturales. Los profesores norteamericanos tenían una actitud más favorable hacia los servicios educacionales de los superdotados y se mostraban partidarios de proporcionar clases especiales. En cambio, los finlandeses postulaban "la misma educación para todos", manifestando reserva hacia clases especiales y teniendo consecuencias negativas frente a este tipo de intervención, ya que la educación de niños talentosos ha sido considerada como elitismo.

Está última mirada también fue encontrada por Chan \& Yuen (2015) en un estudio realizado con estudiantes universitarios que debían tomar un curso electivo sobre educación de talentos. Futuros profesores de educación preescolar, básica y media que hacían parte de esta muestra, consideraron que "todos los estudiantes tienen talentos y que es su función alentar y apoyar a los estudiantes a alcanzar su potencial" ( $p$. 204), pues de lo contrario disminuiría la motivación por aprender a medida que se avanza de curso.

En España, las investigaciones destacan cómo la escasa formación pedagógica ha hecho que la atención a estudiantes con talento, sea insuficiente, que los profesores idealicen a los niños con talento $y$, en consecuencia, sobreestimen las capacidades que estos poseen impactando el proceso de identificación al interior del aula (Fernández, 2002; Medina, 2006). De manera similar, los hallazgos de Kontiyianni et al. (2009) reflejan que aun teniendo estudiantes talentosos - en este caso particular en matemática- los profesores no les dan un trato especial, pues no poseen una real comprensión de las características que los definen como talentosos.

Por otro lado, se ha evidenciado que participar en programas de capacitación en educación de talentos mejora la percepción, actitud y motivación de los profesores, reconociendo que la alta capacidad implica necesidades educativas especiales y atención diferente (Castro, 2005; Fernández, 2002; Tourón, Fernández, \& Reyero, 2005). Aunque, cabe señalar, en el discurso de los profesores se evidencia una dualidad con respecto a quién debe hacerse car- 
go de la educación de niños que presentan talento académico. Algunos tienden a afirman que deben ser integrados al sistema educativo, mientras otros creen que su educación es competencia exclusiva de educadores especiales (Medina, 2006).

A nivel latinoamericano se encuentran algunas investigaciones que reportan las percepciones o concepciones de los profesores acerca de la educación de talentos. El reconocimiento de características cognitivas en los niños con talento es lo más preponderante en el discurso de los profesores (García-Cepero et al., 2012; Flanagan \& Arancibia, 2005; López, Bralic, \& Arancibia, 2002). Por ejemplo López et al. (2002) reportaron en su estudio que las representaciones de los profesores acerca de niños talentosos académicamente se acercan a las definiciones y características planteadas en la literatura, describiéndolos como aquellos que aprenden fácilmente y se encuentran motivados, y cuyos rasgos cognitivos tienen que ver con agilidad, capacidad de relacionar conocimiento, pensamiento científico y creatividad. Dichos resultados son similares a los descritos en la investigación de Olthouse (2014) quien encontró que futuros profesores de educación básica y preescolar presentan tensiones en relación a sus concepciones sobre los estudiantes talentosos predominando la tendencia a creer que ellos destacan dentro de un grupo por su alta capacidad para almacenar y recuperar una gran cantidad de información, en desmedro de la creatividad, la abstracción y motivación.

Aunque también encontramos investigaciones en las que los resultados no son alentadores, ya que el reconocimiento de niños con talento es nulo y la postura frente a su educación es vaga y superficial. Por ejemplo Maia-Pinto \& Fleith (2004) reportaron que profesores de colegios particulares y públicos de enseñanza básica afirmaron que nunca habían tenido estudiantes talentosos en sus salas, además de un conocimiento superficial de la educación del talento, de los procesos de identificación y de las prácticas educacionales acordes a este tipo de estudiantes. Los hallazgos reportados por Fernández (2002) y Medina (2006) irían en esta misma línea.

En relación a cómo se visualiza la práctica educativa con estudiantes con talento, García-Cepero et al. (2012) señalan que los profesores reconocen tener responsabilidad en el trabajo educativo que debiese realizarse con estos estudiantes pese a no tener formación en el área, así como "la necesidad de visibilizar a aquellos que siendo talentosos ocultan su potencial ya sea por timidez o por miedo a la burlas de sus compañeros" (p. 1337). En cuanto al trabajo educativo, relevan el delegar en los niños con talento una función de monitor y apoyo al trabajo docente al interior de la sala, con sus compañeros. Estos resultados son opuestos a los encontrados por Cárdenas (2010), en donde los profesores consideran que los estudiantes con talento, tienen el "éxito asegurado en la sociedad", postulan "la misma educación para todos" para no fomentar el elitismo. Aunque en su discurso también se señaló que el trabajo emocional es suficiente para responder a la formación de estos estudiantes y que de recibir una atención especial debe ser dada por docentes especializados.

Un mayor entendimiento acerca de las concepciones que tienen los profesores de aula regular que no han recibido formación respecto a la educación de talentos nos permitiría tener una mirada más amplia del continuo en el que definimos talento académico; proponer capacitaciones que atiendan a las necesidades reales de formación, considerando las concepciones que los profesores traen con respecto al tema, y, en consecuencia, brindar a los niños con talento oportunidades de aprendizaje de acuerdo a sus condiciones. En particular, el presente estudio fue llevado a cabo para obtener desde el discurso de los profesores de aula regular — sin formación en el tema- cómo conciben y caracterizan a los niños con talento académico. Interesa, por tanto, develar aquellas concepciones de los profesores fruto de las experiencias en su entorno social y que no tienen un sustento teórico o una evidencia empírica. Reconocer dichas concepciones nos acerca a su actuar en la práctica, pues es muy probable que éstas impacten sus acciones y conductas en beneficio o perjuicio de este tipo de estudiante.

\section{Método}

La presente investigación adoptó una metodología cualitativa que aplica los procedimientos metodológicos de la Grounded Theory de Glaser y 
Strauss (Strauss \& Corbin, 1990). Particularmente se consideró que tanto la selección de la muestra, la recolección de los datos y el análisis de los mismos se desarrollaran en forma paralela y no en fases sucesivas, tal como lo plantea esta metodología.

\section{Participantes}

Los participantes en la investigación fueron profesores de segundo ciclo de enseñanza básica de la Región Metropolitana de Chile. El grupo total de profesores estuvo conformado por diez mujeres y tres hombres, con edades comprendidas entre 33 y 63 años (media=53.1; DT=8.2). En su totalidad, todos eran profesores titulados con especialidades en lenguaje, inglés, matemática, física, química, biología, historia y algunos de ellos con estudios de posgrado. Todos los participantes contaban con basta experiencia docente, al menos 15 años de ejercicio de la profesión. Estos pertenecían a establecimientos educativos de diversas dependencias: cuatro pertenecientes a particular subvencionado, cinco a particular pagado y cuatro a establecimientos municipalizados de la Región. Es importante señalar que considerando el objetivo del estudio de caracterizar las concepciones de los profesores en relación al talento académico, se buscó que estos impartieran asignaturas académicas diferentes de las de arte, música y educación física y, además, que no hubiesen recibido previamente capacitación formal en educación de talento.

\section{Instrumentos}

\section{Dilemas}

Se plantearon cuatro situaciones educativas que mostraban distintas posturas de profesores frente a una situación particular en torno a la educación de talentos. El entrevistado debía elegir aquella opción que reflejara su pensamiento o bien establecer cuál sería su opinión frente a la situación presentada en caso de que no estuviese de acuerdo con ninguna de las indicadas. Cabe señalar que estos fueron desarrollados y piloteados previamente a la investigación. El uso de este instrumento, tal como lo señala Pozo
(2006), favorece el acceso a niveles más implícitos de las concepciones de las personas, justamente porque el conflicto está puesto en un tercero, lo que reduce la deseabilidad social.

\section{Entrevistas semiestructuradas}

Para ahondar sobre los significados que poseen los profesores en torno a la educación del talento, se realizaron entrevistas semiestructuradas. Los profesores fueron invitados a establecer un diálogo en el que, a partir de temas-eje, profundizaran sobre sus experiencias y aquellos aspectos que ellos consideraban relevantes en torno a la educación de niños con talento académico. Tal como plantean Strauss \& Corbin (2002), se realizaron preguntas sensibilizadoras (icuál ha sido su experiencia docente con niños talentosos?); teóricas (icree usted que existe alguna relación entre el talento académico y la dependencia del establecimiento educacional?, icuál?); prácticas (si en su sala hay niños con talento, iqué estrategias utiliza usted para trabajar con ellos?; y preguntas guía (iusted cree que el talento es innato o adquirido?, ipor qué?

\section{Análisis de la información}

La codificación y análisis de los datos se realizó siguiendo los procedimientos metodológicos propuestos por la Grounded Theory. Una codificación abierta que permitió realizar un análisis descriptivo de la información y una codificación axial con un análisis relacional. Para asegurar la confiabilidad de la información los datos fueron codificados por tres jueces: una psicóloga, una psicopedagoga y una educadora experta en educación de talentos. Esto permitió la triangulación de la información obteniendo así las categorías conceptuales de las que se desprenden las concepciones que subyacen en el discurso de los profesores participantes.

\section{Procedimiento}

Se obtuvo la autorización de los directores de los colegios para realizar el estudio al interior de sus instituciones, además del consentimiento informado de los propios participantes en el que se explicó 
tanto el objetivo de la investigación como su carácter voluntario, confidencial y anónimo.

\section{Resultados}

A continuación se presentan los hallazgos obtenidos en este estudio, siguiendo el procedimiento de la Grounded Theory. Inicialmente se describen las cinco categorías emergentes identificadas a partir del discurso de los profesores, las cuales reflejan las concepciones que estos tienen en relación a los niños con talento y su educación. Posteriormente se muestra el análisis relacional, identificando tres fenómenos emergentes. Se incluyen extractos literales del discurso de los participantes con el propósito de ejemplificar los análisis realizados.

\section{Análisis descriptivo}

\section{Desarrollo cognitivo, social y emocional como marcadores distintivos en los niños con talento}

Los profesores entrevistados -sin importar la dependencia- visibilizan a los niños talentosos con alta capacidad intelectual, rápidos en su aprendizaje y con motivaciones e intereses particulares:

Son inquietos [los niños talentosos], son habilidosos, son niños que por lo general no te escriben porque te captan todo en clase, trabajan en un nivel más alto, ellos son capaces de relacionar, de comparar, de crear, están sobre el resto del curso. (M, 2012 Agosto, Colegio)

Reconocen que no necesariamente el desempeño cognitivo de sus estudiantes va a la par con su desarrollo social o emocional. En sus palabras: "el niño que es una bala académicamente, que aprende mucho, no por ser tan inteligente va a saber actuar inteligentemente en cada situación que le toque vivir [...]" (M, 2012 Octubre, Colegio).

Asimismo, explicitan que como cualquier niño, ellos enfrentan los mismos desafíos en su desarrollo: "[...] enfrentan los mismos problemas, ellos los solucionan de distinta forma [...]" (M, 2012 Septiembre, Colegio).
Los profesores consideran que no siempre hay asociación directa entre talento y buenas calificaciones: "Yo tengo este año dos chicos que son así [talentosos], (...) de repente tú ves las notas, no lo reflejan, pero tú ves que participan en clase, son bravos, deberían tener puros sietes, pero no lo tienen" (H, 2012 Septiembre, Colegio).

Finalmente los profesores cuentan que si estos niños no son desafiados cognitivamente se aburren en clase: "[...] en términos académicos a un niño que no le enseñan lo suficiente, o que no es exigido con distintos desafíos intelectuales, también se debe aburrir, debe ser una fomedad para él" (M, 2012 Octubre, Colegio).

\section{El origen como factor determinante del talento}

Los profesores reconocen que los niños con talento académico nacen con un potencial genético innato que los diferencia de los demás, pero que requiere de un ambiente que lo estimule y desarrolle para que no se estanque o se pierda. En sus palabras: "[...] porque el niño trae algo, que es su intelecto, sus genes, su potencial, y tiene que haber un ambiente que ayude a estimular esto" (M, 2012 Octubre, Colegio).

En su discurso puntualizan la diferenciación entre ser un niño con talento y ser esforzado. Son las altas capacidades intelectuales en una o varias áreas lo que marca la diferencia entre estos dos grupos de niños “[...] [el talentoso] es talentoso por naturaleza, y si además tiene un medio que lo estimula, más todavía. No me parece que los niños esforzados tengan ese mérito, son esforzados, son metódicos, pero no son talentosos..." (M, 2012 Agosto, Colegio).

\section{Graduación del talento como factor diferenciador}

En general, los profesores en su discurso señalan que existen dos tipos de talento: general y específico. El primero se evidencia en aquellos niños que poseen capacidades superiores en todas las áreas académicas. El segundo se manifiesta en aquellos niños que destacan en un área en particular.

Hay niños talentosos para matemática, otros para arte, otros para historia, en diferentes áreas, ahora 
cuando hay niños casi perfectos [...] en todas las asignaturas, es como el primero del curso, que se destaca en todo, yo creo que ya ahí, el niño tiene talento en muchas áreas, son niños muy responsables también, o sea, el talento se puede dar en un área o en varias [...]. (M, 2012 Septiembre, Colegio)

\section{Ambientes enriquecidos como factores} potenciadores del talento

Los profesores relevan la importancia de la familia como agentes potenciadores del desarrollo del talento. Ambientes familiares que conocen sobre las características y necesidades de sus hijos con talento versus ambientes deprivados no solo económica sino social y emocionalmente potencian o entorpecen el talento presente en sus hijos. En sus palabras:

[...] porque hay veces que el niño trae potencial pero si el ambiente no se preocupa, no hay padres que leen, que le pregunten al niño iqué hiciste hoy día? entonces se van perdiendo. Creo que depende de la combinación de las dos cosas, no es solamente talento, yo creo que tiene que ver con [...] el ambiente de estimulación, de padres que apoyan [...]. (M, 2012 Octubre, Colegio)

También aparece en su discurso el reconocimiento del colegio como un espacio que debe brindar las oportunidades educativas que potencie el talento académico: "[el talento académico] es más estimulado dentro de un grupo familiar, donde se dan espacios para que se desarrolle, y después el colegio al recibirlo está en esta condición de estimularlo más [...]" (M, 2012 Octubre, Colegio).

\section{El sistema educativo homogeniza la educación}

Los profesores coinciden en afirmar que el sistema educativo no promueve el desarrollo del talento académico:

Si tú te fijas todas las clases están atendiendo siempre a buscar que el alumno logre el porcentaje mínimo, el programa ministerial está centrado en el mínimo, en los contenidos mínimos. Y de repente tú tienes niños que sobresalen y se van quedando en el camino porque tú atiendes al mínimo no vas a lo más [...]. (H, 2012 Septiembre, Colegio)

Los profesores también señalan como factor de riesgo la ausencia de capacitación sobre el tema en su formación profesional:

$[\ldots .$.$] y a ti en la carrera no te preparan, yo soy profe-$ sora de media y mi carrera sumamente así cuadrada, física-matemática, icuándo te van a enseñar a diferenciar? [...] Siento que ellos no tienen por qué ser castigados por tener ese talento. (H, 2012 Septiembre, Colegio)

Aun cuando se sienten responsables de brindar una educación de calidad a todos los niños, reconocen que en la práctica dedican mayor tiempo y esfuerzo a aquellos que presentan dificultades: "yo me siento en deuda en mi sala, me hago la lesa. Porque una siempre se preocupa de los malos [estudiantes], los menos talentosos. Pero a los talentosos no me he dedicado como lo he hecho con los más débiles" (M, 2012 Agosto, Colegio).

Finalmente, algunos reconocen el uso de metodologías participativas y entrega de trabajos extra de mayor complejidad a los niños con talento académico: "algunas veces le he dado cosas extra, de trabajos... cuando ellos [niños con talento] están interesados en aprender más [...]" (E4/PS/ D2).

\section{Análisis relacional}

Tres modelos axiales emergen del análisis relacional de este estudio. Estos muestran el rol del profesor en el desarrollo y potenciación del talento académico, el reconocimiento de los niños con talento en el aula y el fenómeno de la educación como una oportunidad de movilidad social.

\section{El reconocimiento de niños con talento académico en el aula}

Las condiciones causales de este primer fenómeno guardan relación con la percepción de los profesores sobre la existencia de niños que presentan caracterís- 
ticas cognitivas, afectivas y sociales diferentes a las de los niños promedio que asisten a su sala (figura 1). Asimismo, las expectativas de logro que poseen los profesores sobre estos niños tanto como el poder brindar un ambiente que estimule y potencie el talento, se constituyen en el conjunto de condiciones que influyen sobre el fenómeno planteado. Los elementos facilitadores reportados por los profesores refieren al reconocimiento de estos niños a través de características cognitivas que los hace sobresalir entre sus pares, la alta motivación por aprender, así como la diferenciación que puede existir al interior de este grupo. Resulta revelador encontrar que los profesores consideran la posibilidad de que existan niños con talento con un bajo rendimiento académico reflejado en sus calificaciones. Como elementos inhibidores de este fenómeno aparecen algunos problemas de adaptación social y dificultades para establecer relaciones interpersonales, además de la conducta de "mimetizarse" adoptada por estos niños al interior de su grupo para no perder su pertenencia a este. La diversidad al interior del grupo de niños con talento se convierte en un obstáculo en el proceso de reconocimiento de aquellas características que permiten diferenciar a estos niños.

Un rol activo por parte del profesor, la validación social del niño con talento al interior de su grupo, la exigencia académica, la asignación de trabajos más desafiantes o complementarios, así como el acompañamiento en cuanto a su desarrollo social y académico se constituyen en las estrategias de acción-interacción tendientes a disminuir los elementos obstaculizadores. Frente al reconocimiento de estos niños al interior del aula, los profesores resaltan (i) la necesidad de recibir capacitación para trabajar más efectivamente con niños con talento académico que les brinde a estos beneficios académicos y sociales, y (ii) la búsqueda de apoyo a nivel institucional para compensar la ausencia de apoyo por parte de la familia.

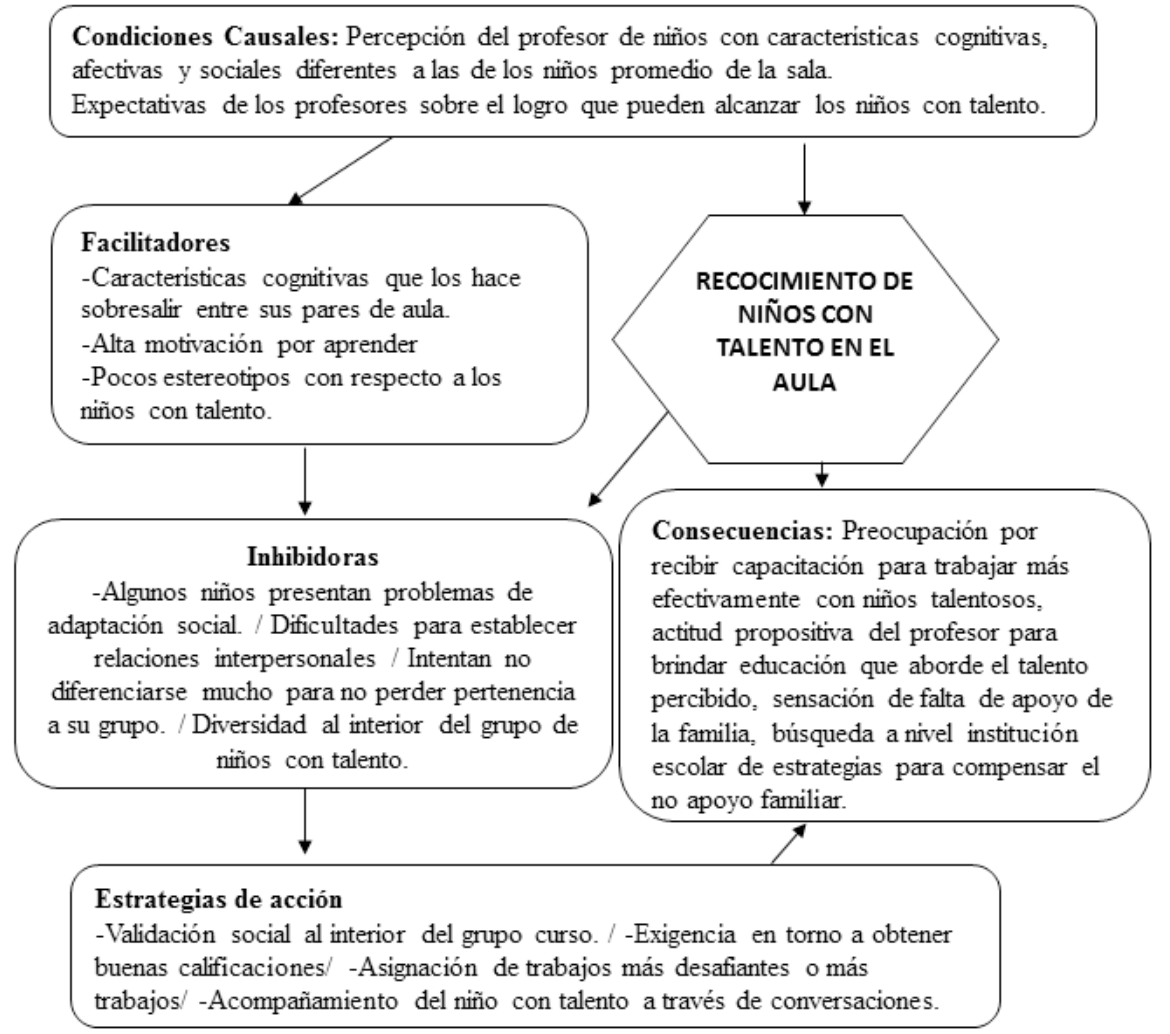

Figura 1. Reconocimiento de los niños con talento en el aula

Fuente: elaboración propia 
Rol del profesor en el desarrollo y potenciación del talento académico

Existe consenso en torno a este Las condiciones causales de este segundo fenómeno se relacionan con el reconocimiento por parte del profesor de niños con talento al interior de sus salas de clase y por tanto, la necesidad de brindar una educación que promueva su desarrollo (figura 2). Una buena disposición para trabajar con todos los niños y la búsqueda de estrategias para ayudar al niño con talento, basadas principalmente en su experiencia e intuición, se constituyen en los elementos facilitadores de este fenómeno. La buena participación en clase y asumir el rol de monitor al interior de su sala se convierten en elementos facilitadores presentes en los estudiantes con talento. La falta de formación docente sobre cómo educarlos y la inequidad en la distribución del tiempo de enseñanza que se otorga a estudiantes con talento versus los estudiantes con dificultades en su aprendizaje se convierten en los elementos inhibidores de este fenómeno. La entrega de material extra a los estudiantes talentosos y la realización de trabajos de investigación, dentro del marco de una metodología participativa, se convierten en las estrategias de acción-interacción más utilizadas por los profesores. Sin embargo, también se identifican estrategias de acción inhibidoras desde el profesor, el alumno y la institución.

Desde esta perspectiva, a nivel de discurso el profesor reconoce las necesidades educativas especiales de los niños con talento, pero en la práctica la dinámica de enseñanza es igual para todos a excepción de los estudiantes con dificultades, pues son estos quienes reciben mayormente estrategias diferenciadas. Como resultado, el alumno talentoso se aburre, participa menos y puede realizar conductas disruptivas. La interrelación de los factores mencionados produce consecuencias que impactan no solo al estudiante, sino también al sistema educativo. Una de ellas es que los estudiantes con talento se desmoti-

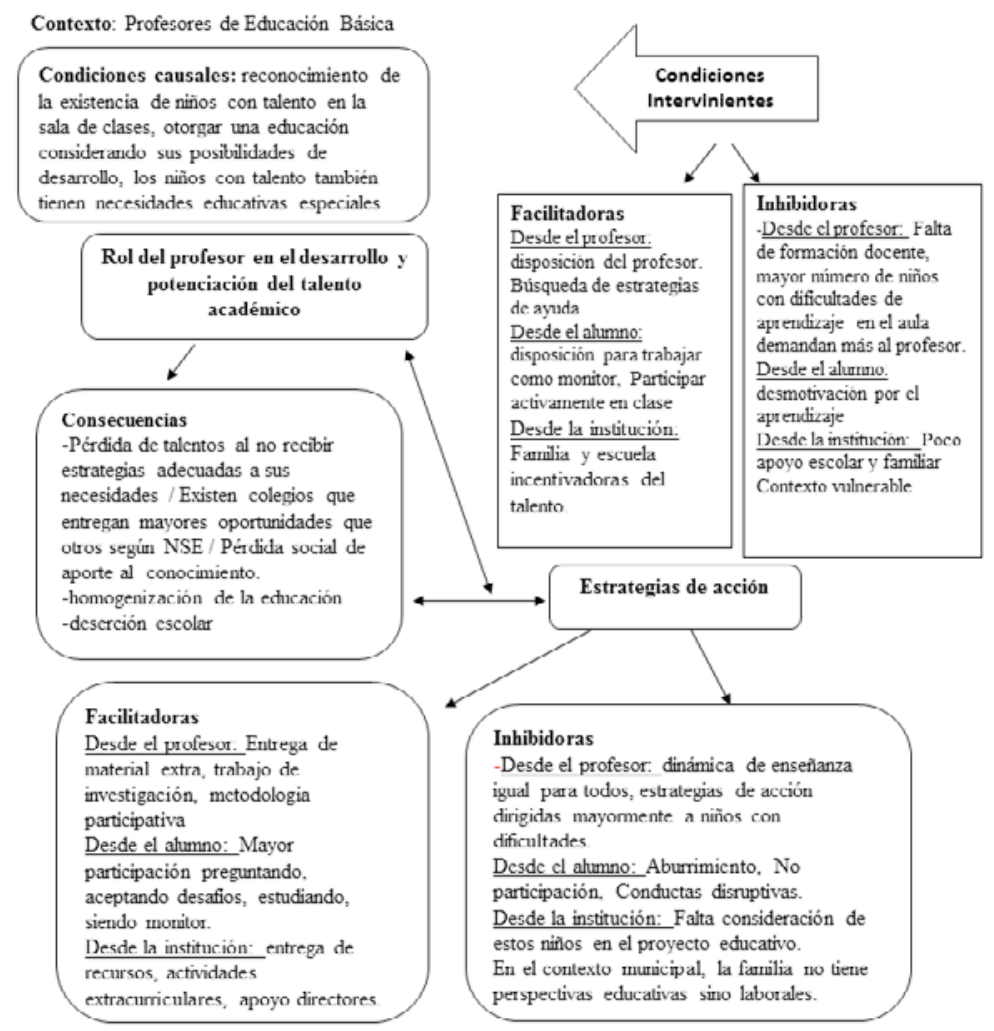

Figura 2. Rol del profesor en el desarrollo y potenciación del talento

Fuente: elaboración propia 
van y, como consecuencia, se pierden talentos por no recibir una educación de acuerdo a sus necesidades. A nivel educativo se observa una homogenización de la educación, la cual se focaliza en enseñar a la media del grupo, sin considerar la atención a la diversidad en todas sus dimensiones. Aspecto relevante cuando partimos de la base de que todos, sin excepción, deberíamos recibir una educación acorde a nuestras necesidades. Finalmente, una consecuencia notoria en los profesores es la necesidad de capacitación para afrontar esta realidad al interior del aula, así como recibir las mismas oportunidades (condiciones-recursos, etc.) en todas las dependencias con el fin de atender a esta diversidad.

\section{La educación como una oportunidad de movilidad social}

Finalmente emerge de los datos este tercer fenómeno para los niños con talento. Las condiciones causales de éste guardan relación con la creencia, por parte de los profesores, de que la educación permite potenciar el talento y que este es el camino que favorece su estimulación (figura 3). Los profesores verbalizan que el talento debe desarrollarse para que no se extinga y que el sistema escolar y la familia juegan un rol fundamental en este proceso. La buena disposición del profesor para trabajar con los niños con talento, la búsqueda de estrategias para promoverlos, la alta motivación del niño por aprender son elementos facilitadores de este fenómeno, y una consecuencia evidente sería la contribución social que estos niños pueden realizar a futuro. Un elemento inhibidor de este fenómeno es la percepción que los profesores tienen acerca del propósito que persigue el sistema educacional. Particularmente ellos consideran que el foco está en que los estudiantes alcancen los contenidos mínimos del currículo, sin contemplar las necesidades educativas especiales del talentoso. Pese a esto, se evidencian algunas estrategias

Contexto: Profesores de Educación Básica

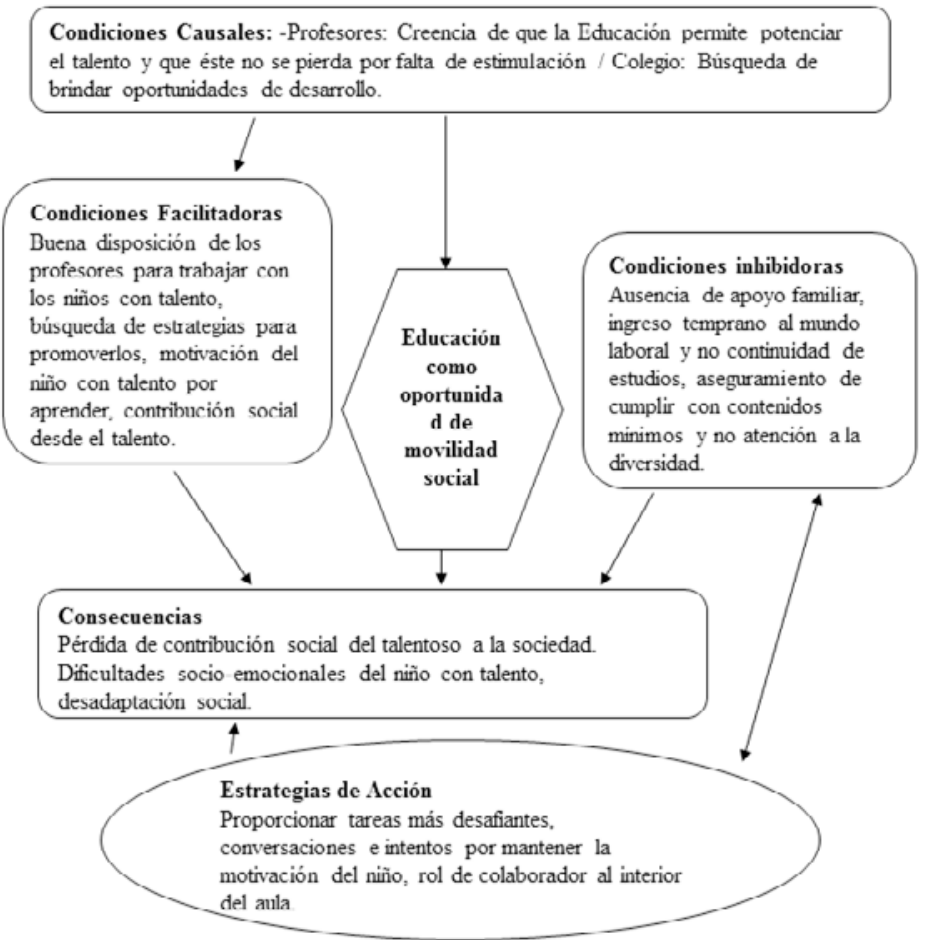

Figura 3: Educación como oportunidad de movilidad social.

Fuente: elaboración propia 
de acción-interacción tendientes a disminuir los elementos obstaculizadores, a saber: la entrega de tareas adicionales y desafiantes, el otorgarles el rol de colaborador con sus pares, así como el tiempo dedicado a conversaciones motivacionales.

Los profesores explicitan en su discurso que los niños con talento se sienten poco apoyados y acompañados en el desarrollo de su talento al no recibir acompañamiento a nivel escolar y familiar, derivando en consecuencias socioemocionales que pueden producirles a futuro dificultades de adaptación social. Los profesores temen la pérdida de la contribución social que puede aportar ese talento, ya que muchos afirman que "socialmente estos niños pueden ser un aporte para retribuirle a la sociedad el don que poseen".

\section{Discusión y conclusiones}

El presente estudio tuvo como propósito caracterizar las concepciones que poseen los profesores de segundo ciclo de educación general básica, acerca de los estudiantes talentosos y la educación del talento. A partir del discurso de los profesores se puede inferir que estos conceptualizan el talento académico dentro de una visión contemporánea en la que se le reconoce como producto de factores personales y de condiciones ambientales y no solamente como producto de una habilidad excepcionalmente alta (Kaufman \& Sternberg, 2008). Este primer hallazgo en la misma línea de los reportados por Chan \& Yuen (2015) y Olthouse (2014) -y a diferencia de los reportados por Flanagan \& Arancibia (2005) -, muestra una conceptualización del talento más inclusiva y menos tradicional (Tapper, 2012) producto de otras características -motivación, compromiso con sus intereses, rapidez en el aprendizaje- más allá de un componente innato.

A nivel más específico, los resultados de este estudio permiten también concluir en relación a los tres fenómenos planteados en el análisis relacional, los cuales se conectan entre sí.

Los profesores de este estudio, reconocen que niños con talento académico en el aula regular tienden a aburrirse si las oportunidades de aprendizaje brindadas no son desafiantes y acordes a sus necesidades
(Benavides et al., 2004; Gallagher, 2003; Chan \& Yuen, 2015). Esta conceptualización recoge contenidos del entorno social que amplían y complementan las ideas y argumentaciones de los profesores, justamente porque, tal como lo señala Rodríguez et al. (1993), las concepciones representan una síntesis de conocimientos obtenidos desde el espacio sociocultural en el que el sujeto se desenvuelve. Contrario a los hallazgos obtenidos en otros estudios (Cardenas, 2010; Castro, 2005; Chung, et al., 2013; Maia-Pinto \& Fleith, 2004; Medina, 2006), en esta investigación los profesores confirman la necesidad de visibilizar a los niños con talento académico al interior del aula, así como la necesidad de contar con capacitaciones que permitan brindarles oportunidades de aprendizaje desafiantes para que se aproveche su motivación por aprender. Explicitar esta carencia en su formación pone en evidencia el reconocimiento de su rol en el desarrollo y potenciación del talento académico. Así, los profesores de este estudio señalan que estudiantes sobresalientes académicamente exigen una formación que favorezca la construcción de clases desafiantes para todos sus estudiantes, incluidos los niños con talento, coincidente con los resultados reportados por Tourón et al. (2005). En su discurso no se evidencia la postura de delegar la responsabilidad educativa en otros profesionales de la educación, sino la de reconocerse como un actor relevante en su educación, hallazgos en la misma línea de los reportados por Medina, 2016; TallentRunnels \& Tirri (1998, citados en Tirri, 2008).

Tal como en el estudio de García-Cepero (2012), los profesores explicitan en su discurso el entregar trabajos extras a los estudiantes que consideran talentosos, aunque reconocen que se guían más por la experiencia e intuición frente al tema, más no por contar con las herramientas pedagógicas que les ayuden a abordar las necesidades de estos estudiantes. Desde esta mirada, los profesores consideran que la educación no homogeneizante es una plataforma de movilidad social solo si responde verdaderamente a las necesidades de los estudiantes talentosos. En relación a lo anterior, se ha demostrado que cuando los docentes participan en capacitaciones y en programas especiales de talentos, no solo cambian su percepción y actitud hacia 
estos niños en forma positiva, sino que aprenden estrategias educativas que se consideran adecuadas en educación de talentos y que pueden ser aplicadas en el aula regular (Cabrera-Murcia, 2011; Chan \& Yuen, 2015, Kontoyianni et al., 2009). Tal como lo avalan las investigaciones, los niños talentosos necesitan de una educación desafiante, que ofrezca un curriculum flexible, complejo y diferenciado que responda a la velocidad, complejidad y profundidad que caracteriza a estos niños -y que ya se explícita en políticas educativas en los países de Europa o de países asiáticos como Hong Kong-, lo cual no se satisface con la sola entrega de trabajo extra (Bralic \& Romagnoli, 2000; López et al., 2002; Sekowski \& Lubianska, 2015; Education Bureau, 2007).

Adicionalmente los resultados muestran que los profesores consideran a la familia, así como a la escuela, ambientes potenciadores del talento. Los hallazgos de este estudio reafirman la necesidad de factores personales tanto como de condiciones ambientales o catalizadores interpersonales que estimulen y motiven el talento (Gagné, 2003). La familia se reconoce como un espacio en el que se deben evidenciar actitudes parentales hacia el aprendizaje y el desarrollo del talento y la escuela como un ambiente enriquecido en el que se brinda un contexto educativo desafiante (López et al., 2002). Nuevamente, al referir a la familia como ambiente potenciador, emerge la concepción de talento en la que se combinan factores personales y ambientales. En su discurso, los profesores explican que el niño que no recibe apoyo familiar difícilmente lo desarrolla. Los factores ambientales, por tanto, influirían en la potenciación del talento. ¿Qué sucede en contextos vulnerables con los niños que poseen un potencial de talento y que el contexto no los promueve? ¿Qué estrategias de acción se implementan a nivel macro para que este capital social no se pierda? ¿Cómo la educación puede ser el timón que permite la movilidad social de estos niños? Reflexionar en torno a estas preguntas y visualizar estrategias de acción que favorezcan el desarrollo del talento académico en los estudiantes que lo poseen, tal como lo afirma García-Huidobro (2005), evitaría consecuencias de tipo social.
Las limitaciones presentes en este estudio permiten vislumbrar futuras líneas de investigación en torno al tema. Una futura línea podría ser realizar un seguimiento a la práctica educativa de estos profesores con el propósito de establecer vínculos entre sus concepciones y su práctica. En esta misma línea, sería relevante evidenciar las concepciones sobre educación de talentos a profesores que ya han recibido capacitación al respecto. Establecer diferencias entre diferentes grupos - con y sin capacitación-nos permitiría mostrar si las concepciones cambian luego de un proceso de formación en este campo de la educación. Asimismo, sería valioso poder recoger desde las familias y la escuela como comunidad educativa aquellas concepciones en la base de la educación de niños con talento con el propósito de avanzar en la implementación de estrategias que verdaderamente ayuden a potenciar el talento en los niños que lo posean. Finalmente, sería relevante poder extraer de las experiencias que ya se están implementando a nivel nacional en relación a la educación de talentos, aquellas prácticas pedagógicas que potencian el talento en los estudiantes y que podrían aplicarse dentro del aula regular.

\section{Referencias}

Benavides, M., Maz, A., Castro, E., \& Blanco, R. (2004). La Educación de niños con talento en Iberoamérica. Santiago, Chile: OREALC \& UNESCO. Recuperado de http://unesdoc.unesco.org/images/0013 /001391/139179s.pdf

Bralic, S., \& Romagnoli, C. (2000). Niños y jóvenes con talentos: Una Educación de Calidad para Todos. Santiago, Chile: Domen Pedagogía.

Cabrera-Murcia, E. P. (2011). Entretejiendo los aprendizajes: desde el programa de perfeccionamiento de la pasantía PENTA UC a la práctica pedagógica. Magis. Revista Internacional de Investigación en Educación, 4(7), 105-120.

Cabrera-Murcia, E. P. (2012). What Must We Know and Know how to do for Implementation during the PENTA UC School Program? A view from the teachers. Universitas Psychologica, 1(3), 815 - 827.

Cárdenas, C. C. (2010). Concepciones de los maestros del instituto técnico Marco Fidel Suárez de la ciu- 
dad de Manizales, frente a las capacidades excepcionales. Investigación. Plumilla Educativa, 7, 25.

Castro, M. (2005). Conocimientos y actitudes de maestros de Educación Infantil, Educación Primaria y estudiantes de Magisterio sobre los niños superdotados intelectualmente. Tesis de Doctorado no publicada, Universidad Complutense de Madrid: Madrid.

Chan, S., \& Yuen, M. (2015). Teachers' beliefs and practices for nurturing creativity in students: Perspectives from teachers of gifted students in Hong Kong. Gifted Education International, 31(3), 200-213.

Chung, D, Kim, Y, Lee, J., \& Park, S. (2013). Differences of Perception on Giftedness between Homeroom Teachers and Teachers of the Gifted. Journal of Gifted/Talented Education, 23(2), 161-175 Recuperado de http://www.koreascience.or.kr/article/ArticleFullRecord.jsp?cn=OJHHBM_2013_ v23n2_161

Education Bureau (2007). Chapter 1: Overview. Retrieved from http://www.edb.gov.hk/index. aspx?nodeID

Fernández, R. (2001). Valoración de los conocimientos, las creencias, y las necesidades del profesorado respecto a la superdotación. Tesis Doctoral no publicada, Facultad de Filosofía y Letras de Universidad de Navarra, España.

Flanagan, A., \& Arancibia, V. (2005). Talento Académico: Un Análisis de la Identificación de Alumnos Talentosos Efectuada por Profesores. Psykhe, 14(1), 121-135. http://dx.doi.org/10.4067/S071822282005000100010

Gallagher, J. (2003). Issues and challenges in the education of gifted students. En N. Colangelo, \& G. A. Davis (Eds.), Handbook of Gifted Education_(3 ${ }^{\text {rd }}$ Ed.) (pp.11-23). Boston: Allyn and Bacon.

Gagné, F. (2003).Transforming Gifts into Talents: The DMGT as a developmental Theory. In N. Colangelo, \& G. A. Davis (Eds.), Handbook of gifted education ( $3^{\text {rd }}$ Ed.) (pp. 60-74). Boston: Allyn and Bacon.

García-Cepero, M. C., Muñoz, E., Proestakis, A., López, C., \& Guzmán, M. (2010). Estudiantes sobresalientes en Establecimientos Educacionales Municipalizados de la Segunda Región._Fundamentos para una política pública para el desarrollo del talento en la escuela. Santiago, Chile: FONIDE - Ministerio de Educación.

García-Cepero, M.C., Proestakis, A., Lillo, A., López, C., \& Guzmán, M. (2012). Caracterización de estudiantes desde sus potencialidades y talentos académicos en la región de Antofagasta, Chile. Universitas Pshychologica, 11(4), 1327-1340.

García-Huidobro, J. (2005). ¿Es necesaria en Chile la educación de talentos? En Penta UC (Ed.), III Seminario Internacional. El modelo PENTA UC para la educación de talentos académicos (pp.14-16). Santiago de Chile.

Jaffri, H. (2012). Conception of giftedness and talent by pre service and in service primary school teacher in Johor, Malaysia: An exploration using a multiphase mixed methods design. Doctoral thesis, Durham University. Recuperado de http:/etheses.dur.ac.uk/3937/

Kaufman, S. B., \& Sternberg, R. J. (2008). Conceptions of giftedness. En S. Pfeiffer (Ed.), Handbook of giftedness in children (pp. 71-91). Tallahassee, FL: Springer.

Kontoyianni, K., Kottou, M., Ioannou, P., Eredotou, M., Christou, C., \& Pittalis, M. (2009). Perceptions on teaching the mathematically gifted. Proceedings of the Sixth Congress of the European Society for Research in Mathematics Education. January 28th - February 1st 2009, Lyon- France.

López, V., Bralic, S., \& Arancibia, V. (2002). Representaciones sociales en torno al talento académico: Estudio cualitativo. Psykhe 11(1), 183-201.

Maia-Pinto, R., \& Fleith, D. (2004). Avaliação das práticas educacionais de um programa de atendimento a Alunos superdotados e talentosos. Psicología Escolar e Educacional, 8(1), 55-66.

Medina, M. (2006). Análisis de las actitudes del profesorado ante la educación de los niños superdotados. Memoria de Doctor en Educación. Universidad Complutense de Madrid: Madrid. Facultad de Educación. Recuperado de http://eprints.ucm.es/ tesis/edu/ucm-t29286.pdf

Ministerio de Educación de Argentina (2006). Ley de Educación Nacional - Ley 26.206. Recuperado de http://secgral.unsl.edu.ar/docs/Ley\%2026206\%20 de\%20Educacion\%20Nacional.pdf

Ministerio de Educación Bolivia (2010). Ley de Educación "Avelino Siñani- Elizardo Pérez" N. 070. 
Recuperado de http://www.oei.es/quipu/bolivia/ Leydla\%20.pdf

Ministerio de Educación Nacional de Colombia (MEN) (2006). Orientaciones para la atención educativa a estudiantes con capacidades o talentos excepcionales. Recuperado de http://www.colombiaaprende.edu.co/html/mediateca/1607/articles-75158_archivo.pdf

Pajares, M. (1992). Teachers Beliefs and Educational Research: Cleaning Up a Messy Construct. Review of Educational Research, 62, 307-332.

Olthouse, J. (2014). How Do Preservice Teachers Conceptualize Giftedness? A Metaphor Analysis. Roeper Review, 36(2), 122-132. 11p. http://dx.doi.org/ 10.1080/02783193.2014.884200.

Pozo, J. (2006). Nuevas formas de pensar la enseñanza y el aprendizaje: Las concepciones de profesores y alumnos. Barcelona: Grao.

Plunkett, M., \& Kronborg, L. (2011). Learning to be a Teacher of the Gifted: The Importance of Examining Opinions and Challenging Misconceptions. Gifted and Talented International, 26(1), 31-46.

Rodríguez López, J. M. (1999). Las teorías implícitas sobre la enseñanza de los profesores en formación antes de las prácticas: el caso de Alicia. Revista de Educación (1), 133-156.

Rodrigo, M., Rodríguez, A., \& Marrero, J. (1993). Las teorías implícitas, una aproximación al conocimiento cotidiano. Madrid: Visor.

SEP (2003). Una Propuesta de Intervención Educativa para Alumnos y Alumnas con Aptitudes Sobresalientes. México. Recuperado de http://basica.sep.gob.mx/dgdgie/cva/sitio/start. php?act $=$ sobresalientes\&sec $=$ ava

Sekowski, A., \& Lubianka, B. (2015). Education of gifted student in Europe. Gifted Education International, 31(3), 73-90.

Sumpter, L., \& Sternevik, E. (2013). Prospective teachers' conceptions of what characterize a gifted student in mathematics. MAVI Mathematical Views 18th Conference (Helsinki, September 12 to September 15). Helsinki, Finlandia. Recuperado de http://urn.kb.se/resolve?urn=urn:nbn:se:du-11941

Strauss, A., \& Corbin, J. (2002). Bases de la investigación cualitativa. Técnicas y procedimientos para desarrollar la teoría fundamentada. Colombia: Universidad de Antioquia.

Tapper, L. (2012). Conceptions of giftedness in a global, modern world: where are we at in Aotearoa New Zealand 2012?. APEX: The New Zealand Journal of Gifted Education, 17(1). Recuperado de http:// www.giftedchildren.org.nz/apex

Tourón, J., Fernández, R., \& Reyero, M. (2002). Actitudes del Profesorado hacia la Superdotación para el Desarrollo de Programas de Formación. Faisca (9), 95-110

Tirri, K. (2008). Who should teach gifted students? Revista Española de Pedagogía (240), 315-324

Vilanova, S., García, M., \& Señoriño, O. (2007). Concepciones acerca del aprendizaje: diseño y validación de un cuestionario para profesores en formación. Revista electrónica de investigación educativa, 2(9) . Recuperado de http://redie.uabc.mx/ vol9no2/contenido-vilanova.html 